\title{
BACKSTAGE: ACERCA DE LAS DECISIONES METODOLÓGICAS EN UN ESTUDIO SOBRE JÓVENES INMIGRANTES Y EMPODERAMIENTO
}

\author{
BACKSTAGE: APPROACH TO METHODOLOGICAL DECISIONS IN A STUDY ON \\ IMMIGRANT YOUTH AND EMPOWERMENT ${ }^{1}$
}

\section{BACKSTAGE: SOBRE AS DECISÕES METODOLÓGICAS NUM ESTUDO SOBRE JOVENS IMIGRANTES E CAPACITAÇÃO}

\author{
Sonia PÁEZ DE LA TORRE \\ Universidad de Girona. Instituto de Investigación Educativa
}

PALABRAS CLAVES:

jóvenes inmigrantes empoderamiento

biograma

Latinoamérica

teoría cultural

Catalunya

\footnotetext{
Fecha de recepción del artículo: 28.11.2017

Fecha de revisión del artículo: 15.111.2017 Fecha de aceptación final: 23.IV.2017
}

RESUMEN: Este artículo forma parte de una investigación en curso que busca conocer los procesos de empoderamiento de jóvenes entre 20 y 34 años originarios del cono sur de América Latina que, tras una experiencia migratoria, viven desde hace más de un año en Catalunya. En la introducción se presentan las fuentes de la investigación y se recuperan las aportaciones que brindaron los estudios sobre la inmigración latinoamericana en España. Esta revisión revela la necesidad de actualizar las perspectivas y las metodologías en el área de interés; sirve también para comprender el planteo de este estudio: el objetivo, el recorte del universo, la hipótesis y el encuadre teórico-metodológico sustentado en el concepto de empoderamiento y en las propuestas de Pierre Bourdieu. En un segundo momento se describen las primeras actividades que se llevaron a cabo antes de ingresar al campo y que permitieron planear las estrategias metodológicas adecuadas. Luego se detalla el diseño, el proceso de validación y la finalidad de la herramienta que se creó en base a la actualización de la técnica de los biogramas propuesta por Theodore Abel (1947). Se recolectaron 50 "Biogramas A"

CONTACTAR CON LOS AUTORES: Sonia Páez de la Torre. Universidad de Girona. Instituto de Investigación EducativaDepartamento de Pedagogía. Plaça Sant Domènec, 9, 17004 Girona. Desp. 044 -Tel +34-972-419664. soniapaezdelatorre@gmail.com. FINANCIACIÓN: Este trabajo se lleva adelante gracias al apoyo de la Secretaria d'Universitats i Recerca del Departament d'Economia i Coneixement de la Generalitat de Catalunya y al financiamiento del Fondo Social Europeo de la Unión Europea. Está vinculado también al Proyecto HEBE: El empoderamiento de los jóvenes: análisis de los momentos, espacios y procesos que contribuyen al empoderamiento juvenil. MINECO - Programa Estatal de 1+D+l Retos de la Sociedad 2013. Ref.: EDU2O13-42979-R. Dr. Pere Soler Masó (IP) (Universitat de Girona).

*Este trabajo obtuvo el "IV Premio Jóvenes Investigadores en Pedagogía Social", otrogado por la Sociedad Iberoamericana de Pedagogía Social en el XXIX Seminario Interuniversitario de Pedagogía Social, celebrado del 14 al 16 de Septiembre de 2016 en Girona. 


\begin{tabular}{|c|c|}
\hline & $\begin{array}{l}\text { con los que se buscaba principalmente conocer el volumen y la estructura del capital eco- } \\
\text { nómico, social y cultural que tenían estos jóvenes inmigrantes en sus territorios de origen y } \\
\text { algunos rasgos sobre la transformación que sufrieron los mismos en la sociedad de acogida. } \\
\text { En función a una serie de variables predeterminadas se consigue seleccionar } 24 \text { participantes } \\
\text { a quienes se les aplicará un "Biograma B". El objetivo de esta aportación, es dar cuenta del } \\
\text { método de investigación implementado para garantizar tanto el éxito del proceso de recogida } \\
\text { de datos como la fiabilidad y la calidad de la información y presentar las primeras aportacio- } \\
\text { nes que todo este proceso ha brindado. }\end{array}$ \\
\hline $\begin{array}{l}\text { KEY WORDS: } \\
\text { young immigrants } \\
\text { empowerment } \\
\text { biograma } \\
\text { Latin America } \\
\text { cultural theory } \\
\text { Catalonia }\end{array}$ & $\begin{array}{l}\text { ABSTRACT: This article is part of a research in progress that seeks to understand the em- } \\
\text { powerment processes of young people aged 2O-34 from Latin America's Southern Cone that } \\
\text { after a migratory experience are living in Catalunya for more than a year. The introduction } \\
\text { presents the research's sources and recovers the contributions provided by immigration stud- } \\
\text { ies on Latin Americans in Spain. This review reveals the need to update the perspectives and } \\
\text { methodologies in the area of interest; it is also used to understand the approach of this study: } \\
\text { the goal, trimming of the universe, the hypothesis and methodological framing supporting the } \\
\text { empowerment concept and the proposals of Pierre Bourdieu. Secondly, the first activities } \\
\text { conducted prior to entering the field and that allowed to plan appropriate methodological } \\
\text { strategies are described. Then it is detailed the design, validation process and purpose of } \\
\text { the tool that was created based on the update of the biogramas' technique proposed by } \\
\text { Theodore Abel (1947). } 50 \text { "Biogramas A" were collected, to know the volume and structure of } \\
\text { the economic, social and cultural capital that these young immigrants had in their territories } \\
\text { of origin and some features on the transformation suffered at their arrival. According to a } \\
\text { series of predetermined variables, } 24 \text { participants are selected to apply a "Biogram B". The } \\
\text { objective of this contribution is to deal with the research method implemented in order to } \\
\text { ensure both the success of the data collection process as well as the reliability and quality of } \\
\text { the information and to present the first contributions obtained through this process. }\end{array}$ \\
\hline $\begin{array}{l}\text { Palavras-chave: } \\
\text { jovens imigrantes } \\
\text { empoderamento } \\
\text { biograma } \\
\text { América Latina } \\
\text { teoria cultural } \\
\text { Catalunha }\end{array}$ & $\begin{array}{l}\text { RESUMO: Este artigo é parte de uma investigação que visa compreender os processos de } \\
\text { capacitação dos jovens entre } 20 \text { e } 34 \text {, originarios do sul da América Latina, que passaram por } \\
\text { uma experiência de migração, que vivem na Catalunya há mais de um ano. Na introdução as } \\
\text { fontes de pesquisa e suas contribuições são apresentados a partir dos estudos sobre a imi- } \\
\text { gração latino-americana na Espanha. Esta avaliação revela a necessidade de atualizar as pers- } \\
\text { pectivas e metodologias na área de interesse; também serve para entender a proposta deste } \\
\text { estudo como: o objetivo, amostragem, pressupostos teóricos e a estrutura metodológica, sus- } \\
\text { tentada pelo conceito de empoderamento propostas por Pierre Bourdieu. Em um segundo } \\
\text { momento são apresentadas as primeiras atividades que foram realizadas antes de adentrar } \\
\text { no campo de estudo e a descrição das estratégias metodológicas adequadas, em seguida, o } \\
\text { desenho, o processo de validação e o propósito da ferramenta que foi criada com base na } \\
\text { atualização técnica do "biograma", proposto por Theodore Abel (1947). Foram coletadas } 50 \\
\text { "Biogramas A", no qual procurou saber o volume e estrutura de capital econômico, social e } \\
\text { cultural que tinha esses jovens imigrantes nos seus territórios de origem e algumas caracte- } \\
\text { rísticas sobre a transformação sofrida por eles na sociedade que o receberam. Em função de } \\
\text { uma série de variáveis predeterminadas foi selecionados } 24 \text { participantes que foram aplica- } \\
\text { dos um "Biograma B". O objetivo desta contribuição é explicar o método de pesquisa e sua } \\
\text { implementação para garantir o sucesso da coleta de dados; a confiabilidade e a qualidade da } \\
\text { informação bem apresentar as primeiras contribuiçães do proceso da pesquisa. }\end{array}$ \\
\hline
\end{tabular}

\section{Introducción}

El origen de este estudio se vincula a la experiencia migratoria personal y se sitúa en el marco del Proyecto $H E B E$, en el que surgen las principales preguntas de investigación: ¿̇en qué medida los jóvenes originarios del cono sur latinoamericano residentes en Catalunya han podido potenciar, desarrollar y aumentar sus capacidades, sus aprendizajes, sus conocimientos y su autonomía?, ¿En qué espacios, momentos y de qué modo expresan se configura su proceso de empoderamiento?, ¿Qué rol ha cumplido el contexto social (los adultos, las instituciones, las asociaciones)?, ¿Qué oportunidades les ha brindado este entorno centrado en el adulto? ¿Han buscado alternativas de empoderamiento en otros espacios centrados en los jóvenes?

Un análisis inicial de la bibliografía disponible revela la necesidad de actualizar los estudios, las metodologías y las perspectivas de análisis que se han utilizado hasta ahora para abordar los procesos de migración en diferentes lugares del país (los estudios se han centrado principalmente en los contextos de Madrid y Barcelona). Una gran cantidad de estos trabajos han analizado el fenómeno de la inmigración latinoamericana en España que comienza a mediados de los ‘ 80 y va incrementando a finales de los ' 90 y comienzos del 2000. Por ejemplo, han sido frecuentes los 
estudios que tratan de describir las trayectorias educativas: la escuela como primer espacio de socialización en el que niños y adolescentes entran en contacto con las prácticas culturales de la sociedad receptora (Marin, Feixa \& Nin, 2013). En este caso, se trata de valorar como lo educativo imprime ciertas características en los itinerarios de este tipo de jóvenes. También se ha investigado el proceso de inserción de los jóvenes inmigrantes en el mercado laboral (Cachón, 2011), comparando las actividades que desarrollan y las condiciones contractuales que tienen respecto a los autóctonos. Otro tipo de estudios, como el desarrollado por Retis García (2010), han puesto en evidencia la representación negativa mostrada por la prensa sobre este grupo social al visibilizar, desde una perspectiva altamente dramática, problemáticas protagonizadas por jóvenes inmigrantes en el espacio público.

Como consecuencia de la evolución de los procesos migratorios y del conocimiento aportado por algunos de estos estudios, la situación de estos jóvenes ha entrado a formar parte del debate de las políticas públicas de manera explícita a nivel nacional, autonómico y local. Periódicamente se recogen datos, se analiza y se estudia la realidad de este grupo y se proponen estrategias para mejorar su integración o para prevenir su exclusión social (Jiménez, 2010). Son también numerosas las reflexiones teóricas y estudios cualitativos que insisten en la necesidad de favorecer la participación cívica de los jóvenes inmigrantes, como uno de los retos fundamentales para lograr la igualdad de oportunidades y la homogeneidad en el tejido social (vale la pena citar la compilación de estudios coordinada por López Sala y Cachón, 2007, los estudios de Romaní y Feixa, 2012, o los de Eseverri Mayer, 2015, entre muchos otros).

\subsection{El proceso de empoderamiento de los jóvenes del cono sur latinoamericanos que viven en Catalunya: trayectorias en transición hacia la vida adulta}

El término "Latinoamérica" hace referencia a un amplísimo espacio geográfico -Centro América, Caribe y América del Sur- y con ello a países, prácticas culturales, realidades y procesos sociales, históricos y económicos muy diferentes. Por ello, en nuestro trabajo limitamos nuestro interés a indagar sobre las trayectorias de jóvenes y jóvenes adultos originarios del cono sur latinoamericano que tras un proceso de migración viven en Catalunya, entendiendo a los jóvenes como sujetos activos. Las decisiones que ellos van tomando les permiten construir su propio itinerario y dibujar su propia biografía en un determinado escenario social -cambiante y complejo-. En este proceso van teniendo, paulatinamente, un lugar en la sociedad. Esta propuesta teórica hace hincapié en el individuo, sin dejar de subrayar la incidencia del contexto en las modalidades y complejidades de las transiciones. Se entiende entonces, que no es lo mismo transitar la juventud en una sociedad que ofrece pocos caminos, con finales más o menos previsibles -como ocurría en las sociedades industriales o como ocurre en muchos países de Latinoamérica, señalados por las desigualdades y la escasa movilidad social-, que realizar la transición a la vida adulta en el marco del capitalismo informacional, en el que existen una gran diversidad de modalidades de transición, caracterizadas por las contradicciones, las incertidumbres y los riesgos (Casal, 2011).

Dentro de este marco geográfico, merecen atención especial países como Chile, Uruguay y Argentina, en tanto existe una semejanza entre los procesos históricos, políticos, económicos y sociales seguidos por los tres países, más allá de las muchas diferencias que puedan existir. Los tres territorios fueron colonias españolas, por lo tanto la cultura precolombina se ha fusionado con la occidental y la lengua oficial es el castellano; una vez independizados, re-abrieron las puertas a las distintitas oleadas migratorias europeas que cruzaron el océano en épocas de guerras, posguerras, crisis y dictaduras; inversamente, cuando Latinoamérica fue sacudida por los golpes de estado (1970 en adelante), los exiliados políticos argentinos, chilenos y uruguayos buscaron refugio en Europa y principalmente en España, favorecidos por la lengua y, en algunos casos, por lazos que aún se mantenían con familiares que se habían quedado en el viejo continente; un último rasgo que tienen en común estos tres espacios geográficos está relacionado con un similar progreso socio-económico, sobre todo en comparación al resto de Latinoamérica².

Es por ello que nuestro estudio está centrado en los y las jóvenes -y jóvenes adultos- de 20 a 34 años, originarios del cono sur de América Latina que están efectuando o han efectuado su transición hacia la vida adulta en el contexto catalán y se encuentran: a) estudiando; b) trabajando; c) estudiando y trabajando; d) ni estudiando ni trabajando. La hipótesis de investigación busca constatar si la migración, durante el período de la juventud, puede ser pensada como un proceso de empoderamiento en sí mismo: se trata de una oportunidad para incrementar las capacidades del individuo, la auto-confianza y el desarrollo de un gran abanico de habilidades sociales. El cambio de espacio sociocultural implica el inicio de un proceso de superación de dificultades, de 
crecimiento y fortalecimiento personal, entre otros desafíos. A su vez, en la sociedad de acogida existen una serie de ofertas educativas, laborales, culturales y sociales a la que este conjunto de jóvenes intentará acceder, de acuerdo a sus intereses y expectativas de vida. Las posibilidades que dicho contexto les ofrezca o restrinja, determinadas por un marco político y legal específico, condicionarán su relación con el entorno, sus modalidades de integración y de empoderamiento.

La teoría del capital cultural, propuesta por Pierre Bourdieu (Bourdieu, 1986; 1999; Bourdieu \& Passeron, 1967; entre otros) guía las preguntas que subyacen a este planteamiento y permite delimitar el objetivo específico: conocer cuál es la relación que existe entre el capital económico, social, cultural y simbólico de estos jóvenes y las posibilidades de empoderamiento que les ofrece el contexto social en el que se insertan.

Sobre todo resulta valiosa la aportación que la perspectiva del empoderamiento brindará al trabajo en tanto esta investigación no aspira a enfocar ni a la inmigración, ni a la juventud como una problemática social. Comas (2011) señala que la juventud como categoría social es sistemáticamente puesta en duda tanto desde los discursos académicos como desde las retóricas y prácticas políticas. Se niega la existencia de una identidad juvenil, de una clase social juvenil y, en contraposición, se insiste en la condición común que tiene este grupo de estar a la "espera de" ser adultos, ser ciudadanos activos, tener un empleo regular y estable, un domicilio, una familia propia, etc. La paradoja, como bien subraya Comas (2011), es que, el hecho de que los jóvenes sean definidos por "lo que no son", da cuenta de que "son" una categoría social y por lo tanto susceptible de tener una identidad positiva. Es justamente por ello que los estudios de juventud y los proyectos que buscan intervenir en la realidad juvenil se han apropiado de la noción de empoderamiento (Jennings et al., 2009)

Excede los límites de este trabajo abordar la complejidad del concepto de empoderamiento, tan impreciso y a su vez tan ampliamente difundido en la actualidad. Se trata de una noción que pone en evidencia el desequilibrio de poder que hay entre unos grupos sociales y otros; por ello, es interesante tener en cuenta que en los últimos 15 años ha comenzado a aplicarse al campo de los estudios de juventud. Algunas de las reflexiones desarrolladas por el equipo HEBE en torno a este concepto, han sido recientemente publicadas por Úcar Martínez, Jiménez-Morales, Soler- Masó y Trilla Bernet (2016). Desde la perspectiva de la pedagogía social, hacen un doble esfuerzo por explicar cómo y con qué sentido se ha usado esta noción en el ámbito académico; y por comprender la aplicación y la intervención que se hizo en el campo de la juventud en los últimos años.

Una de las propuestas centrales radica en comprender al empoderamiento como un proceso, como "el resultado de una interacción, de una negociación, hacia un grado mayor o menor, entre la capacidad o capacidades de una persona, grupo o comunidad, y las opciones que le o les ofrecen los contextos físicos y socioculturales en que desarrollan sus vidas" (Úcar Martínez, et al., 2016: 5). Los autores hacen referencia a dos dimensiones: la personal -en la que juegan un papel central las propias capacidades, las experiencias, los aprendizajes adquiridos a lo largo del itinerario vital-; y la contextual, es decir, el entorno sociocultural y las posibilidades y oportunidades que ofrece o niega para actuar. En este sentido, consideran que las personas jóvenes se empoderan mediante procesos que les permiten superar ciertas situaciones, adquiriendo de este modo competencias y aprendizajes para participar, intervenir y transformar el contexto en el que viven, desde una posición activa y emancipadora.

El presente estudio se inspira y se nutre de estas reflexiones teóricas, pero planea realizar más aportaciones para seguir pensando este concepto en función a los resultados a los que se arriben. Inicialmente se propone entender al empoderamiento como un proceso que se desarrolla poniendo en diálogo las capacidades personales de los jóvenes originarios del cono sur latinoamericano, el reconocimiento que hace la sociedad de acogida de dichos capitales y los recursos que -de acuerdo a ese reconocimiento- les ofrece la sociedad. En la medida en que la relación dialéctica entre la esfera individual y la social se efectúe satisfactoriamente, sea cercana y positiva, los y las jóvenes serán capaces de decidir y actuar sobre su propia vida; empoderados y activos, podrán también efectuar trasformaciones y aportaciones a la comunidad de pertenencia (Zimmerman et al. 2010).

El objetivo de este artículo es exponer las estrategias metodológicas que posibilitaron la puesta en marcha del trabajo de campo. Para garantizar tanto el éxito del proceso de recogida de datos como la fiabilidad y la calidad de la información, se desarrollaron una serie de actividades previas que develaron la dificultad para arribar al colectivo de jóvenes inmigrantes del cono sur latinoamericano que reside en Catalunya.

La revisión de la perspectiva de análisis de Pierre Bourdieu y de la teoría metodológica relacionada al problema planteado, permitió rescatar y actualizar la técnica de biogramas que propuso en 1947 Theodore Abel. Interesa explicar cómo se diseñó esta herramienta, el proceso de validación al que fue sometida, su utilidad y la finalidad para 
este estudio. Posteriormente se definen las variables que se tuvieron en cuenta para seleccionar los perfiles de los participantes y se explica cómo se ha procedido en la aplicación de uno de los biogramas. En un último apartado se reflexiona sobre cómo avanzar en este estudio.

\section{Metodología. Del cuarto oscuro del investigador al montaje de la escena}

\subsection{Backstage: notas para iluminar el campo de estudio}

El trabajo del investigador se asemeja al de los viejos profesionales de la fotografía analógica. La tarea consiste en reunir una serie de productos sensibles a la luz, en pasar varias horas encerrado en un cuarto oscuro para lograr, con paciencia y delicadeza, una imagen de alta calidad. Para el caso del investigador, resulta necesario realizar ciertas actividades importantes que, aunque puede que luego formen parte del backstage, definen la calidad de los resultados.

Para iluminar el campo que interesa a este estudio, una de las primeras tareas consistió en mapear los recursos, redes y organizaciones sociales creadas por el colectivo de inmigrantes originarios del cono sur latinoamericano que viven en Catalunya. Se entabló contacto con los Consulados, a quienes se les explicó las características del proyecto de investigación y se les solicitó acceder a toda información que pudiera llegar a ser útil: noticias sobre las asociaciones existentes en la comunidad; y datos (cantidad, edad, sexo, año de llegada, lugar de procedencia y de residencia, distribución geográfica, etc.) sobre los y las jóvenes originarios de Argentina, Chile o Uruguay que viven en el territorio.

Paralelamente se rastreó por internet a las agrupaciones uruguayas, chilenas y argentinas de la comunidad. La recolección de correos electrónicos, direcciones, teléfonos y páginas web, permitió crear una base de datos. Se les envió un mensaje presentándoles también el proyecto, sus objetivos y el blog que se inauguró con la idea de fundar, justamente, un canal de diálogo con los y las jóvenes, cuyas realidades se quiere conocer ${ }^{3}$. Además de pedirles información general sobre el colectivo de interés también se les propuso un encuentro, pues interesaba saber cómo funciona el centro: cuándo se fundó, qué actividades se desarrollan y qué relación tiene con los y las jóvenes.

Una tercera actividad consistió en buscar páginas, grupos y comunidades de Facebook llevadas adelante por chilenos, argentinos y uruguayos que viven en Catalunya y sistematizar algunos datos que pudieran servir -tipo de página, cantidad de miembros, finalidad de la misma-. Una vez que aceptaron la solicitud de amistad se siguió con el protocolo: presentación del proyecto y del blog. Aunque no se trabajó recolectando datos de estas páginas porque funcionan caprichosamente y porque las fuentes no son del todo confiables, sí se hizo un seguimiento continuo de las mismas para estar al caso de las actividades que se realizan, de las necesidades y preocupaciones que emergen en estos espacios.

\subsection{Luz, cámara y iacción!: algunas pistas}

La información que los Consulados brindaron fue muy general. El de Argentina y Chile estimaron el número aproximado de conciudadanos que viven en Catalunya, el de Uruguay en cambio no disponía de dicha información. El Consulado uruguayo recomendó localizar a las asociaciones en una sección de la web oficial; el de Chile prefirió reenviar el pedido, por cuenta propia, a sus asociaciones y el de Argentina no hizo referencia al tema en su respuesta.

Se descubrieron 24 agrupaciones fundadas por inmigrantes originarios del cono sur latinoamericano en la comunidad ${ }^{4}$. Las respuestas de estos centros tampoco fueron masivas. Se concretaron 6 encuentros con los representantes de las organizaciones que, de un modo u otro, intentan no naufragar en los resabios de la crisis y el masivo retorno de los compatriotas. Las entrevistas han permitido alumbrar algunos aspectos sobre la realidad de los y las jóvenes, pistas que guiaron las decisiones metodológicas de este trabajo. Asimismo, inauguraron un contacto más directo con el grupo de interés, ya que se recibió invitaciones para participar de eventos a los que se asistió y en los que se pudo contactar con participantes que colaboraron con este estudio ${ }^{5}$.

Del seguimiento de las páginas de Facebook, se advierte que algunas son muy activas, otras están abandonadas o tienen pocos usuarios. Los integrantes no residen necesariamente en Catalunya. Circula información de todo tipo: fiestas, lugares para conocer, temas sobre trámites y papeles -requisitos, dificultades o dudas para solicitar el NIE, datos sobre dónde aprender catalán, información respecto a cómo revalidar los títulos-, se consulta sobre los precios y el costo de vida, aparecen ofertas y pedidos de servicios; se cuelgan noticias de allá y de acá. Este espacio virtual funcionó como un eficaz canal para difundir el blog y recibir feedback sobre los post, para estar al caso de encuentros que se realizan, para compartir los instrumentos metodológicos con los que se recolectaron datos y para contactar con participantes que formaron parte del estudio. 


\subsection{El montaje de la escena: estrategia metodológica}

Los primeros acercamientos al campo han sido enriquecidos con la revisión de la teoría propuesta por Pierre Bourdieu (referenciada más arriba) y las aplicaciones metodológicas que de esas herramientas han realizado numerosas investigaciones en: a) el ámbito de la sociología de la juventud y en el de educación (Martín Criado, 2004; Cairns, 2015; entre otros); y b) en el de estudios de inmigración (Sayad, 2010; Garzón, 2006, 2010; Erel, 2010; Nowicka, 2015; entre otros).

La evidencia obtenida en el primer acercamiento al campo mostró que no existen vías institucionales concretas para arribar al colectivo de jóvenes que interesa estudiar. La manera de llegar a posibles participantes, parece ser a través de las redes informales y los contactos que la investigadora pudiera tener. Las actividades previas, las lecturas y las reflexiones de este proceso, permitieron rescatar y actualizar la técnica de biogramas que propuso en 1947, Theodore Abel:

Los biogramas son relatos de vida que un investigador demanda a miembros de un determinado grupo social. El grupo es seleccionado de acuerdo al problema que el investigador quiere estudiar. La demanda incluye directivas específicas sobre el contenido que debe tener el relato. Cada participante, consciente $\circ$ inconscientemente, seleccionará algunos episodios de su experiencia; las instrucciones que se dan hacen que se focalice en ciertos aspectos que deben ser incluidos y descriptos en detalle. En este sentido el investigador se asegura de obtener el máximo de información relevante de un problema, dejando, además, que se escriba libremente. (Abel, 1947: 114) ${ }^{6}$

Abel explica que este instrumento es valioso en tanto permite recoger información masivamente; facilita el estudio del cambio social; y la detección de patrones que muestren el comportamiento de un grupo social específico. Indica además la importancia de hacer explícita en la demanda del relato, la contribución que se hace con el mismo a un proyecto científico. Apunta, por último, que los biogramas no son un método autosuficiente, sino que el término debe ser usado preferentemente en plural: un solo relato no basta para obtener pistas sobre un determinado colectivo.

Este estudio no pretende ser representativo del grupo social al que intenta conocer, por ello no plantea una recolección de datos "masiva" para conformar una "muestra". En cambio, sí interesa obtener información general sobre las biografías de los y las jóvenes originarios del cono sur latinoamericano que viven el Catalunya. Biograma, explica Feixa (2006: 12), es un término que se elige no en función a su etimología sino por su analogía lingüística "si el habla de cada individuo no se entiende sin la gramática de su lengua, la historia de vida resulta ininteligible sin referirse al biograma que comparte con otros miembros de su grupo social".

\subsection{Laboratorio: el diseño de los instrumentos}

A la luz de estas consideraciones, se diseñó una primera herramienta: el Biograma A. Se trata de un formulario que consta de 53 preguntas, mayoritariamente de opción múltiple, aunque también se incluyeron espacios para que el participante pueda narrar libremente. Con la aplicación de este instrumento se buscaba en primer lugar, conocer el volumen y la estructura del capital económico, social y cultural que tenían estos jóvenes inmigrantes en sus territorios de origen y algunos rasgos sobre la transformación que sufrieron los mismos en la sociedad de acogida. La información biográfica que se demandaba se estructuró en base al eje temporal allá/acá, para favorecer la reconstrucción del proceso y del proyecto inmigratorio del participante. Una segunda finalidad radicaba en detectar aquellas trayectorias en las que indagar con mayor profundidad, mediante la aplicación de un Biograma B y una entrevista semi-estructurada.

El Biograma B consta de 39 preguntas, también de selección múltiple y en algunos casos abierta. Esta herramienta se configuró para profundizar en algunos aspectos puntuales sobre la conformación del capital social, cultural y económico de los jóvenes, centrando la atención en el eje temporal del presente. El propósito es, por un lado, conocer algunos aspectos sobre la vida social del participante en la sociedad de acogida, sus expectativas y proyecciones vitales a futuro; por otro, seleccionar con un mejor criterio, los perfiles más ricos y diversos para llevar adelante la entrevista semi-estructurada.

Las respuestas obtenidas en los Biogramas $A$ y $B$, constituyen el punto de partida de dicha entrevista. Se le propone así al participante un encuentro cara a cara en el que pueda narrar su recorrido como inmigrante de una manera más profunda, más extendida. Todo esta información y la posibilidad de un segundo encuentro personal si fuera necesario, posibilitarían la reconstrucción de relatos de vida.

Tanto el Biograma A como el B, están precedidos por un breve texto que explica los objetivos del estudio, su relación con el proyecto HEBE y el nombre de las instituciones que respaldan el 
trabajo. Además, se garantiza el anonimato de los participantes, la protección de los datos y el uso de la información estrictamente vinculada a la investigación. Para el caso de la entrevista, se incluye un consentimiento informado firmado por el participante para grabar el encuentro.

\subsection{Prueba y error: el proceso de validación}

Para garantizar la calidad de los resultados, los instrumentos fueron sometidos a un proceso de validación. Se consultó a un total de seis expertos y se realizaron cuatro pruebas pilotos. En un primer momento, las herramientas se presentaron y se pusieron en discusión en un seminario de investigación.

Las devoluciones obtenidas permitieron iniciar una segunda fase, en la que se consultó a dos profesionales que están vinculados con temáticas de juventud e inmigración y que, además, cuentan con la vivencia personal de haber emigrado por un periodo largo durante su itinerario formativo.

En esta etapa surgieron dudas sobre cómo indagar en la homologación o convalidación de los títulos universitarios extranjeros. Concretamente lo que interesa saber es si los inmigrantes que poseen dicho capital cultural, cuentan con el reconocimiento institucional de la sociedad de acogida; y si les ha permitido acceder a un puesto de trabajo relacionado con la formación que hicieron en su país de origen. La información que ofrece la página del Ministerio de Educación es confusa; entonces se realizó la consulta a un quinto experto, que además de ser investigador en formación es de origen argentino y reside hace 7 años en Catalunya, por lo que tenía experiencia en dicho trámite. Su perspectiva permitió re-formular la enunciación de una manera más sencilla. Se decidió que, en todo caso, este aspecto sería un tema a indagar con mayor profundidad en la entrevista semi-estructurada.

En una tercera fase se solicitó la colaboración de 4 jóvenes inmigrantes que viven en Catalunya para que realizaran una prueba piloto. Las devoluciones fueron positivas: subrayaron que los instrumentos eran simples, claros y fáciles; que las opciones múltiples eran suficientes y el lenguaje comprensible. Para concluir todo el proceso, los formularios fueron revisados por una sexta investigadora; esta vez se optó por alguien de origen catalán, para verificar si el estudio era accesible para alguien ajeno a la experiencia migratoria. Las observaciones fueron satisfactorias; se consideró entonces que ya se podía comenzar con la recolección de datos ${ }^{7}$.

\section{Resultados y discusión. El etalonaje.}

\subsection{Puesta en marcha: variables y aplicación del Biograma A}

La estrategia metodológica, se diseñó pensando en recolectar 50 Biogramas $A$, calculando un margen, para poder seleccionar 24 perfiles. Transversalmente se consideró que un aporte para el campo de estudio en cuestión, radicaba en contactar con jóvenes que no hayan arribado siendo niños, puesto que en ese caso la complejidad del proceso migratorio -la sociabilización, integración, etc.-, la atravesaron probablemente los padres. Para establecer un criterio se determinó que aquellos que emigraron con menos de 12 años (es decir, los que se integraron en el sistema educativo, cultural y social español-catalán en los niveles primarios) no serían casos a tener en cuenta en este estudio.

Teniendo en cuenta el objetivo específico de esta investigación, se estableció que lo central en estos 24 perfiles sería encontrar jóvenes que tengan ocupaciones diversas, es decir que: estudien; trabajen; estudien y trabajen; no estudien, no trabajen o tengan trabajos precarizados. Se priorizó esta variable, porque se entiende que la conformación de los capitales está relacionada con la ocupación de los participantes. Orientativamente se comprende por "estudiante" a aquellos jóvenes que se encuentren realizando estudios formales (bachilleratos, ciclos formativos, estudiantes universitarios, de máster y de doctorado); por "trabajo" a quienes Ileven desarrollando actividades rentadas hace por lo menos 6 meses y tengan un contrato de entre 15 y 20 horas semanales. $\mathrm{Si}$ el trabajo es los fines de semana o solo por la temporada, se considera que el joven tiene un trabajo precarizado. En el caso de que "estudie y trabaje", la dimensión "estudio" admite solo a los ciclos de educación formal y la de "trabajo", a aquellos contratos de menos de 15-20 horas por semana. En la condición de "N/N/TP", entran aquellos jóvenes que están con trabajos precarizados (que lleven menos de 6 meses en el ámbito laboral, que describan una situación inestable, cambiante o temporal); o que estén realizando estudios terciarios, o aquellos que expliciten estar buscando trabajo, o que manifiesten estar en una situación poco favorable.

Una segunda variable que se consideró importante es la de país de origen (se pensó en intentar una representación equitativa de jóvenes nacidos en Argentina, Chile y Uruguay). La tercera y cuarta variable que se tuvo en cuenta, es la equidad de 
género y la diversidad etaria (tratando de tener en cuenta que el rango delimitado es muy amplio y que seguramente la situación sería diferente para aquellos entre 20 y 24 , para los que tienen entre 25 y 29 y para los que tienen entre 30 y 34 ).

La recepción de Biogramas A estuvo abierta durante 2 semanas aproximadamente (desde el O6 de Julio de 2016 hasta el 21 de Julio de 2016). Se solicitó colaboración en las páginas de Facebook a las que se les hace seguimiento, se les escribió a los representantes de las asociaciones con los que ya se había contactado, se escribió correos personales a aquellas personas que son originarias del cono sur latinoamericano y se pidió que colaboren re-enviando el formulario a sus conocidos (utilizando así, la técnica que se conoce como bola de nieve).

En este tiempo se obtuvieron un total de 50 respuestas de las que se eliminan dos: una que correspondía a una persona que tenía 43 años; la segunda porque la persona vive en Chile. Por lo tanto, se trabajó en base a 48 respuestas: 28 corresponden a argentinos (20 mujeres y 8 hombres); 11 a uruguayos (4 mujeres y 7 hombres); y 9 a chilenos (7 mujeres y 2 hombres).

De los 48: 6 se dedican solamente a estudiar (son todas mujeres); 18 solamente trabajan (7 hombres y 11 mujeres), 15 estudian y trabajan ( 7 hombres y 8 mujeres) y 8 están buscando trabajo, cobrando el paro o tienen trabajos precarizados (hace menos de 6 meses, menos de 20 hs por semana; algunos en negro, otros tienen problemas para trabajar debido a su situación legal).

\subsection{Procedimientos metodológicos: la selección de los perfiles}

A partir de la información brindada por los 48 participantes se procedió a realizar una primera limpieza, intentando por un lado la máxima representación de las variables planteadas pero siguiendo, por otro lado, criterios de fiabilidad y calidad de la información. Una primera lectura de los datos permitió descartar a: a) aquellos participantes que dijeron no estar interesados en un encuentro para seguir participando en esta investigación; b) aquellos cuyos datos eran dudosos, confusos o poco fiables; c) aquellos que emigraron siendo muy chicos y ya llevan un largo tiempo viviendo en España. Se excluyeron un total de 10 participantes: 4 de Chile, 3 de Argentina, 2 de Uruguay; 1 hombre y 9 mujeres; 4 estudian y trabajan, 3 trabajan; 2 estudian. De este modo los perfiles que quedaron pre-seleccionados son 38 .

La segunda fase de selección, se sustentó en la variable "ocupación", que es la que se había señalado como la más importante. Se intentó tener participantes que representen las cuatro condiciones:

- En cuanto a aquellos que en el Biograma A dijeron que sólo estaban estudiando, se los conservó a todos porque sólo había 3 casos de los 6 que se pretendían tener idealmente. Además, estaban representados jóvenes originarios de los tres países del cono sur.

- El mismo procedimiento se aplicó para el caso de aquellos que contestaron que en ese momento tenían trabajos precarizados o que estaban buscando trabajo. Se mantuvieron a los 6 participantes que había (3 jóvenes uruguayos y 3 argentinos; no hubo casos con esta variable entre los participantes del grupo chileno).

- En cuanto a los que respondieron que su ocupación es trabajar, se conservó el único caso que había de un joven originario de Uruguay y los dos casos que habían de jóvenes originarios de Chile. Para la elección de las respuestas de jóvenes originarios de Argentina, en el que había más participantes, se tuvo en cuenta la equidad de género y de manera complementaria la edad. Se tomaron 2 casos más de lo que idealmente se había pensado, puesto que el grupo de jóvenes que estudian tenía menos de lo pensado.

- De manera similar se procedió con los que respondieron que estudian y trabajan, se reservaron los tres casos de jóvenes originarios de Uruguay y dos de chilenos. Para escoger a los jóvenes originarios de argentina, en el que había más respuestas, se tuvo en cuenta la equidad de género y de manera complementaria la edad de los participantes. Se tomó 1 caso más en el grupo de argentinos y 1 caso más en el grupo de los jóvenes uruguayos, de lo que idealmente se había pensado, puesto que el grupo de jóvenes que "estudian" había menos de lo pensado.

La selección de los 24 perfiles a quienes se les solicitaría que completaran el Biograma B quedó conformada por: 3 jóvenes que estudian (las tres mujeres, una originaria de Uruguay, otra de Argentina y otra de Chile); 7 jóvenes que trabajan (4 mujeres y 3 hombres; 1 uno originario de Uruguay, 4 de Argentina y 2 de Chile); 8 jóvenes que estudian y trabajan ( 6 hombres y 2 mujeres; 3 originarios de Uruguay, 3 de Argentina y 2 de Chile); 6 jóvenes que tienen trabajos precarizados o que no están estudiando o trabajando ( 3 hombres y 3 mujeres; 3 originarios de Uruguay, 3 de Argentina y ninguno de Chile). 


\subsection{Aportaciones finales: ihacia delante!}

Desde el ámbito de la pedagogía social las metodologías de la sociología cualitativa y de la antropología cultural han sido útiles, entre otras cosas, para conocer las transiciones de las personas a lo largo del tiempo, los cambios que en ella se van generando y que, entramados a “(...) otras variables socioambientales como el estatus socioeconómico, los contextos étnicos y parentales, religiosos y socioculturales, configuran ese discurrir vital" (García, 1995:42). El planteo y el uso de estas técnicas, que en educación se han dado a conocer bajo el genérico de "metodologías etnográficas o etnografía educativa", ponen el acento en la mirada, la voz y la experiencia de los individuos (Goetz y LeCompte, 1988). Esto significa resignar a la posibilidad de realizar generalizaciones; a cambio se obtiene la profundidad en los datos y se abre un espacio propicio para el auto-análisis de los participantes, brindando así protagonismo y empoderamiento a las minorías de una sociedad.

En este artículo se ha presentado la primera aportación de este estudio, correspondiente a la fase en la que se encuentra esta investigación , que aunque bien puede considerarse parte del Backstage, resulta fundamental para garantizar la calidad de los resultados. Para avanzar hacia nuevos resultados, es necesario aplicar el Biograma $\mathrm{B}$ a los 24 jóvenes seleccionados e indagar en el estado actual del capital social, cultural y económico; en sus procesos de integración social; en sus expectativas y proyecciones a futuro. Luego se procederá a seleccionar 12 perfiles representativos en cuanto a la diversidad de trayectorias y a la conformación de capitales, con quienes entablar un encuentro cara a cara.

\section{Bibliografía}

Abel, T. (1947). The nature and use of biograms. American Journal of Sociology, 57, 111-118.

Bourdieu, P. (1986). The forms of capital. En Richardson, J. (ed.). Handbook of theory for the sociology of education. Greenwood Publishing Group.

Bourdieu, P. (1999). La miseria del mundo (Vol. 1). España: Ediciones Ákal.

Bourdieu, P., \& Passeron, J.-C. (1967). Los estudiantes y la cultura. (3era ed.). Buenos Aires: Editorial Labor.

Cachón, L. (2011). Immigración en España: Del Mercado de Trabajo a la Plena Ciudadanía (Immigration in Spain: From Labour Market to Full Citizenship). Oñati Socio-Legal Series, 1 (3).

Cairns, D. (2015). Learning to Fly: Entering the Youth Mobility Field and Habitus in Ireland and Portugal. En Costa, C. y Murphy, M. (eds.), Bourdieu, Habitus and Social Research. The art of application (pp. 111-125). Palgrave Macmillan UK.

Casal, J. (2011). "Las rupturas del acceso a la vida adulta: Juventud, cambio generacional y vínculo social”. Comunicación presentada en el Seminario Internacional Juventud, cambio generacional y vínculo social. México: UNAM.

Comas, D. A. (2011). ¿Por qué son necesarias las políticas de juventud? Revista de Estudios de Juventud (94) 11-26.

Erel, U. (2010). Migrating Cultural Capital: Bourdieu in MigrationStudies. Sociology, 44 (4), 642-660. Retrieved from http://www.jstor.org/stable/42857433.

Eseverri, C. (2015). Jóvenes sin vínculos. El papel de las estructuras intermedias en un espacio urbano desfavorecido. Revista Española de Investigaciones Sociológicas, 150:23-40.

Feixa, C. (2006). La imaginación autobiográfica. Periferia: revista de recerca i formació en antropologia, 5, 01-44.

García, A. V. M. (1995). Fundamentación teórica y uso de las técnicas historias y relatos de vida como técnicas de investigación en pedagogía social (pp. 41-60) Salamanca: Ediciones Universidas de Salamanca.

Garzon, L. (2006). Trayectorias e integración de la inmigración argentina y ecuatoriana en Barcelona y Milano. Tesis doctoral. Universidad Autónoma de Barcelona. Retrieved from http://ddd.uab.cat/pub/tesis/2006/tdx-0216107-162125/ lggldel.pdf.

Garzon, L. (2010). Migración y movilidad social: argentinos y ecuatorianos entre las "Américas" y las "Europas". Septiembre. IACCHOS - Institute for Analysis of Change in History and Contemporary Societies.

Goetz, J.P., \& LeCompete, M.D. (1998). Etnografía y diseño cualitativo en investigación educativa. Madrid: Morata.

Jennings, L.B, Parra-Medina, D., Hilfinger Messias, D.K., \& Mc Loughlin, K: Hacia una teoría social crítica del empoderamiento de la juventud (2009). En Checkoway, B.N.; Gutiérrez, L.M. (eds) Teoría y práctica de la participación juvenil y el cambio comunitario. Barcelona: GRRAO.

Jiménez-Ramírez, M. (2010). La «juventud inmigrante» en España: Complejidad de una categoría discursiva a debate. Revista Latinoamericana de Ciencias Sociales, Niñez y Juventud, 8 (1), 363-391.

López, A \& Cachón, L. (coord.) (2007). Juventud e Inmigración. Desafíos para la Participación y para la Integración. Dirección General de Juventud de la Conserjería de Empleo y Asuntos Sociales del Gobierno de Canarias.

Marin, J. O., Feixa, C. y Nin, R. (2013). Jóvenes inmigrados en Lleida-Cataluña, España: transiciones escolares y laborales en un contexto de crisis. Revista Latinoamericana de Ciencias Sociales, Niñez y Juventud, 11 (2), 493-514. 
Martin, E. (2004). De la reproducción al campo escolar. En Martin Criado, E. Alonso, L. E, Moreno Pestaña, J.L. Pierre Bourdieu: las herramientas del sociólogo. Madrid: Editorial Fundamentos.

Nowicka, M. (2015) Habitus: its transformation and transfer through cultural encounters in migration. En Costa, C. Murphy, M. (eds.) Bourdieu, habitus and social research. The art of application (pp. 93-110). UK: Macmillan Palgrave.

Retis, J., \& García, P. (2010). Jóvenes inmigrantes latinoamericanos en la prensa española: Narrativas mediáticas de la alteridad: el caso de las violencias urbanas. Revista mexicana de ciencias políticas y sociales, 52 (209), 135-161.

Romaní, O., \& Feixa, C. (2012). Being heard or being seen. En Fangen, K., Johansson, T. y Hammarén, N. (eds). Young migrants. Exclusion and belonging in Europe. London: Palgrave macmillan.

Sayad, A. (2010). La doble ausencia. De las ilusiones del emigrado a los padecimientos del inmigrado. Barcelona: Anthropos.

Úcar, X., Jiménez-Morales, M., Soler Masó, P., \& Trilla, J. (2016). Exploring the conceptualization and research of empowerment in the field of youth, International Journal of Adolescence and Youth, DOI: http://dx.doi.org/10.1080/0 2673843.2016.1209120.

Zimmerman, M. A., Stewart, S. E., Morrel-Samuels, S., Franzen, S., \& Reischl, T. M. (2010). Youth empowerment solutions for peaceful communities: combining theory and practice in a community-level violence prevention curriculum. SAGE publications.

\section{CÓMO CITAR ESTE ARTÍCULO}

Paéz de la Torre, S. (2017). Backstage: acerca de las decisiones metodológicas en un estudio sobre jóvenes inmigrantes y empoderamiento. Pedagogía Social. Revista Interuniversitaria, 30, 205-214. DOI: 10.7179/PSRI_2017.30.14.

\section{DIRECCIÓN COMPLETA DE LOS AUTORES}

Sonia Páez de la Torre: Investigadora en Formación (Becaria FI de la AGAUR). Universidad de Girona.Instituto de Investigación Educativa- Departamento de Pedagogía. Plaça Sant Domènec, 9, 17004 Girona. Desp. 044 -Tel +34-972-419664. soniapaezdelatorre@gmail.com

\section{PERFIL ACADÉMICO}

Sonia Páez de la Torre: Licenciada en letras por la Universidad Nacional de Tucumán de Argentina, (2010), Magister en Juventud y Sociedad por la Universidad de Girona de España (2014). En el 2015 la Agencia de Gestión de Ayudas Universitarias y de Investigación, junto con el Fondo Social Europeo, le otorgaron la Beca Fl para realizar sus estudios de doctorado en la escuela de Educación de la Universidad de Girona. Su investigación se centra principalmente en juventud, inmigración y educación. Forma parte del Instituto de Investigación en Educación (IRE) y desarrolla sus actividades desde el Departamento de Pedagogía de la Universidad de Girona. Es miembro del Grupo de Investigación en Políticas, programas y servicios educativos y socioculturales; y colaboradora en el Proyecto de Investigación CIUNT del Instituto de Investigaciones Lingüísticas y Literarias Hispanoamericanas de la Universidad Nacional de Tucumán de Argentina. https:// fronterasindomitas.wordpress.com/. 